\title{
Pregnancy and Loneliness: The Therapeutic Value of 3D/4D Ultrasound
}

\author{
Ulrich Honemeyer ${ }^{1,3}$, Asim Kurjak ${ }^{2,3}$ \\ ${ }^{1}$ Consultant Obstetrics and Gynecology, Fetal Medicine and Genetic Center DHCC, Dubai, UAE \\ ${ }^{2}$ Zagreb University, Zagreb, Croatia \\ ${ }^{3}$ Dubrovnik International University “Libertas” (DIU), Dubrovnik, Croatia \\ Email: dr.ulrich.ho@hotmail.com
}

Received 13 December 2013; revised 11 January 2014; accepted 5 February 2014

Copyright @ 2014 by authors and Scientific Research Publishing Inc.

This work is licensed under the Creative Commons Attribution International License (CC BY).

http://creativecommons.org/licenses/by/4.0/

(c) () Open Access

\begin{abstract}
The fetal sensual and motoric equipment and its successful development within the maternal organism on the one hand, and the harmonic psycho-physic transformation and maturation of the woman into a mother on the other hand, are linked in dialectic inter-dependence. We describe situations in which the harmony of this process is disturbed. Visualization of the fetus in 3D and real time 3D (4D) can influence parental phantasies of the unborn, improve compromised bonding, or create bonding between fetus and (foster)-parents, and support understanding and even acceptance of fetal pathology.
\end{abstract}

\section{Keywords}

3D/4D Ultrasound, Bonding, Maternal-Fetal Attachment, Frozen Mother, Fetal Akinesia-Deformation Sequence

\section{Introduction}

Hitherto, the prevailing conception in public and scientific circles was that fetal life resembled a state of sedation mediated by high levels of placental progesterone and neuro-inhibitors in the fetus, and that sensory systems and central processing of external stimuli were probably not adequately developed to perceive for example pain (Mellor, 2007).

With the advent of 4D- or real time 3D-ultrasound however, straightforward visualization of the fetal face, body, and of fetal movements had become possible. The human eye can differentiate single images up to a frame rate of $12 \mathrm{~Hz}$. Above this frame rate, serial images are perceived as continuous movement. Presently, peak frame rates of high end ultrasound machines can reach $20 \mathrm{~Hz}$, with still acceptable image quality. This enables 
real-time observation of even small facial twitches and has opened the "doors of perception" of an abundant variety of fetal behavior. The use of 4D ultrasound in all three trimesters has boosted our knowledge about fetal behavior. Old scientific conceptions of prenatal existence have to be discarded in view of 4D ultrasound revelations of fetal life. Scientific analysis of fetal behavioral patterns mediated by 4D ultrasound contradicts assumptions of continuous fetal sedation during pregnancy. Observations of a multitude of behavioral features of the unborn similar to those of the newborn have proved continuity of fetal and neonatal behavior. Besides these new insights in fetal behavior, recent research has made substantial contributions to our knowledge about the developmental timelines of fetal sensory systems: initiated with the somesthetic/proprioceptive systems (tactile/ thermo-reception) on the face, palms, and soles at around 7 weeks gestational age (GA), progressing brainstem maturation enables the vestibular system at around 15 weeks. The oral/nasal chemoreception starts to function at the same time, before the auditory system develops from 20 weeks onwards, followed by the arrangement of the visual system (Lecanuet, 2002). As a result of all these new findings, the question of fetal awareness was up for discussion. The developing proprioceptive "inner world" of the fetus and his gradual opening up to external sensual stimuli find their representations in the "primary awareness" of the fetal brainstem. Primary awareness can be understood as a compound of pre-competences for fetal-maternal attachment.

\section{Discussion}

The fetal motoric and sensual equipment and its successful development within the maternal organism on the one hand, and the harmonic psycho-physic transformation and maturation of the woman into a mother on the other hand, are linked in dialectic inter-dependence. This is the beginning of a lifelong dialogue between the individual and the world, or as A. Clark (2008) phrased it: "The human organisms, and the external world, are a coupled and unified cognitive system" (Clark, 2008). The sensual interface between fetus and mother has many variables: vestibulo-cochleal, chemosensory, olfactory-gustatory, auditory, tactile, psycho-vegetative, endocrinological, and bio-rhythmic stimuli are continuously changing the fetal environment. It is postulated that the fetus memorizes such sensual experiences. Long-term memory requires a functional limbic system and diencephalon (specifically, the hippocampus, amygdala, anterior and mediodorsal thalamic nuclei, and mamillary nuclei); these structures are already well developed and functioning at full term gestation. There is for instance evidence that the human fetus has the ability to detect and record odor information afforded by the mother through her diet (Schaal, 2000). Several studies in animals have demonstrated the persistence of intrauterine acquisition of odor preferences until weaning or adult age (Smotherman, 1991; Bilko, 1994). According to B. Schaal and other researchers, this raises the possibility that intake of flavor-rich food, drinks, of tobacco, alcohol, or other addictive substances, may have latent and deferred effects on the behavior of the offspring (Kirstein, 1997; Kandel, 1994). The morphological development of the inner ear-Cochlea—is completed at 20 weeks. Auditory evoked potentials in premature born fetuses can be registered from 24 weeks: vibro-acustic stimulation at this time produces a fetal motor response. Exposure to voice, music, and meaningful sounds after 30 weeks enables the configuration of tono-topic columns on the outer surface of the auditory cortex of the temporal lobe, a process called "tuning". The first in-vivo evidence of fetal hearing was delivered by non-invasive magneto encephalography (MEG) brain imaging at 38 weeks (Moore, 2007; Graven, 2008). It is known that the fetus recognizes the mother's voice from prenatal exposure and is able to discriminate between different speech sounds in utero. This may have implications for development of language, for recognition of the mother and father, and development of attachment between offspring and parents (Lecanuet, 1993). Visual evoked responses were measured in fetuses from 28 weeks' gestation onwards (Eswaran, 2002).

The Swedish developmental psychologist Claes von Hofsten summarized the development of such fetal pre-competencies elegantly: "Emerging action capabilities are shaped by the subject's interactions with the environment. Perception, cognition, and motivation develop at the interface between neural processes and actions. They are a function of both and arise from the dynamic interaction between the brain, the body, and the outside world" (von Hofsten, 2009). There is more new evidence that such action capabilities develop already early intrauterine. Umberto Castiello and coworkers of the research group around Vittorio Gallese at Parma University found that, starting from the 14th week of gestation, twin fetuses plan and execute movements specifically aimed at the co-twin. For the purpose of this study they analyzed the abdominal four-dimensional ultrasound video sequences of 10 fetuses each recorded for 20 minutes. The video recordings were then digitized through special software allowing off-line kinematic analysis of hand movements. Their study results lead to the assumption “....as fetal behavioral patterns directly reflect developmental and maturational processes of the fetal 
central nervous system, it might be advanced that social patterns might represent early markers of the appearance of developmental disorders affecting the social dimension of behavior” (Castiello, 2010).

Rarefication of fetal or maternal stimuli at the sensual interface between fetus and mother can theoretically result in disturbance of the dialectic inter-dependence between fetal and maternal development during pregnancy. We will call this loneliness in pregnancy. Loneliness in fetal-maternal relation can have its cause either in fetal or in maternal "absence". A biological model for fetal loneliness could be the "frozen mother" i.e. pregnancy in mothers with apallic syndrome, and as biological model for fetal absence, we refer to our recent case of "fetal akinesia-deformation sequence".

\section{3D/4D Ultrasound and Bonding}

The relationship between mother, father, and their child unfolds with the beginning of pregnancy. This early emotional attachment between is rooted deeply in ontogeny, and its intensity becomes obvious in tragic situations of intrauterine fetal death (IUFD) or fetal demise during childbirth. According to Cranley (1981), both physical development of the fetus and transformation of a woman into a mother happen during the 9 months of gestation: "Integral to that development is the consideration of the woman's identity, her role identity, the identity of her developing fetus, and perhaps most important, the relationship between herself and her fetus" (Cranley, 1981). Furthermore, a significant correlation between prenatal and postnatal attachment is long known and clearly proven by research (Muller, 1996; Fleming, 1988). "Bonding”, generally refers to the parent's tie to the infant and is thought to be initiated in the first hours or days of postnatal life. Bonding in connection with 3D/4D ultrasound during pregnancy however occurs prenatally (Klaus, 1995). With 3D/4D ultrasound, the visual experience of the fetus as an independent being already early in pregnancy is likely to influence parental-fetal relationship and to initiate bonding at a much earlier point in fetal development (Stormer, 2003). Both, realities and fantasies regarding the developing fetus influence qualities and strength of prenatal "bonding" or, as synonymously used, of "maternal-fetal attachment" (MFA). In a review of twenty-two studies on maternal-fetal attachment since 2000 by J.L. Alhusen (2008), “...five studies examined how the use of ultrasound impacted maternal-fetal attachment. The majority of the studies examined the differences in levels of MFA when a three-dimensional (3D) or four-dimensional (4D) ultrasound was incorporated, while one study sought to evaluate the impact of ultrasound consultation on MFA. The majority of the studies did not find increased MFA in those women undergoing 3D or 4D ultrasound; however, Boukydis et al.'s (2006) study, which added a component of ultrasound consultation, noted increased MFA in the consultation group. There was also evidence in those studies that measured MFA prior to the performance of any type of ultrasound that MFA was increased based upon having an ultrasound (Righetti, Dell’Avanzo, Grigio, \& Nicolini, 2005).” (Alhusen, 2008). Alhusen states in her review that "The length of time spent performing an ultrasound, expertise of the technician, opportunity to ask questions, and amount of information provided to patients are all important considerations when assessing the influence of ultrasound on MFA. Specifically, one would expect a longer duration of viewing one's baby and a greater opportunity to ask questions pertaining to the ultrasound would influence the overall experience of an ultrasound” (Alhusen, 2008) (Figures 1-5).

\section{Fetal Loneliness}

Esmaeilzadeh and others (Univ. of Heidelberg, Germany, 2010) conducted a systematic literature review for relevant citations of maternal brain death (BD) in pregnancy, and found between 1982 and 2010, 19 case reports and 1 case series. They summarized the 30 reported cases of extended maternal life support after BD with pregnancy and the resulting neonatal outcomes. A non-traumatic brain injury was the cause of BD in 26 of 30 mothers. The maternal mean age at the time of BD was 26.5 years. The mean gestational age at the time of BD and the mean gestational age at delivery were 22 and 29.5 weeks, respectively. The mean duration of maternal support after was 38.3 days (range, 2 - 107 days).Twelve viable infants were born and survived the neonatal period. A cesarean section was the mode of delivery in all cases which resulted in live-born fetuses Not every infant was sufficiently followed to determine the long-term effects of prolonged maternal life support. Postnatal follow-up up to 24 months was available only for six infants. All of them developed normally and apparently had no problems related to their exceptional intrauterine circumstances until 24 months (Esmaeilzadeh, 2010). 3D/4D ultrasound as bonding mediator in this situation should target the caregivers after childbirth, father or foster parents of this child of a "frozen mother". To our best knowledge, there are no scientific reports about the use of 3D/4D 


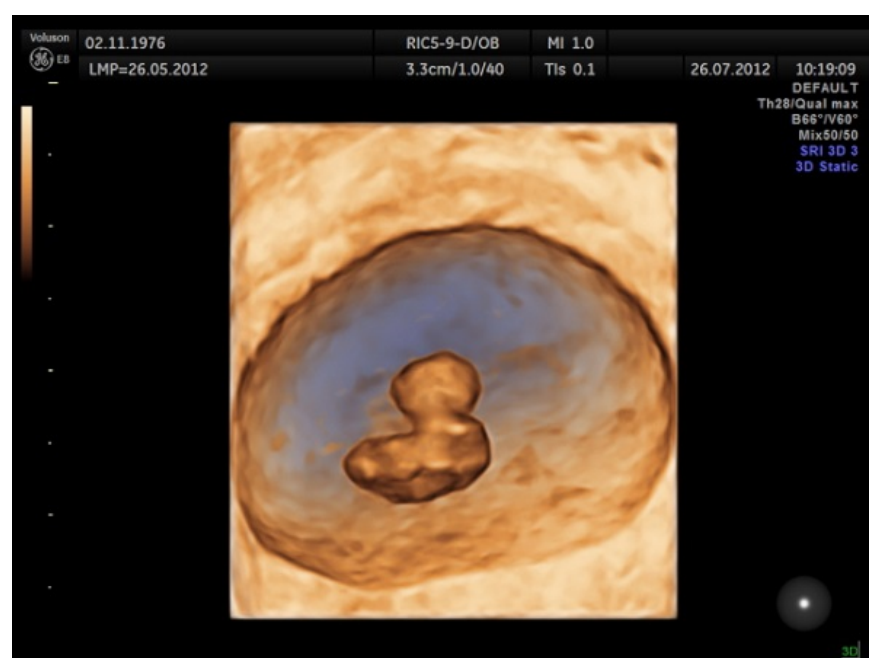

Figure 1. Embryo at seven weeks post menstruationem (pm).

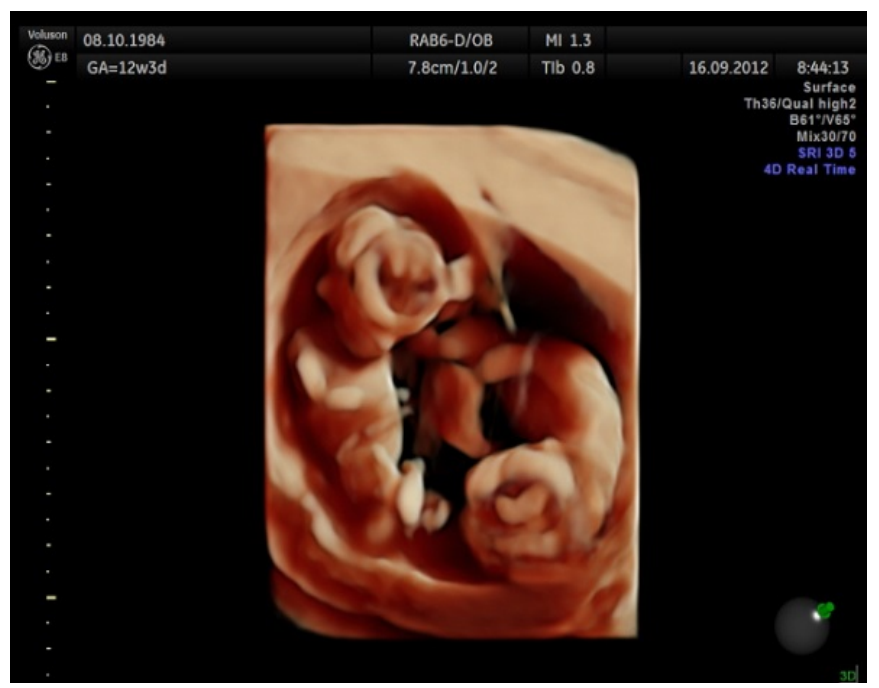

Figure 2. Dichorionic twins at 12 weeks pm.

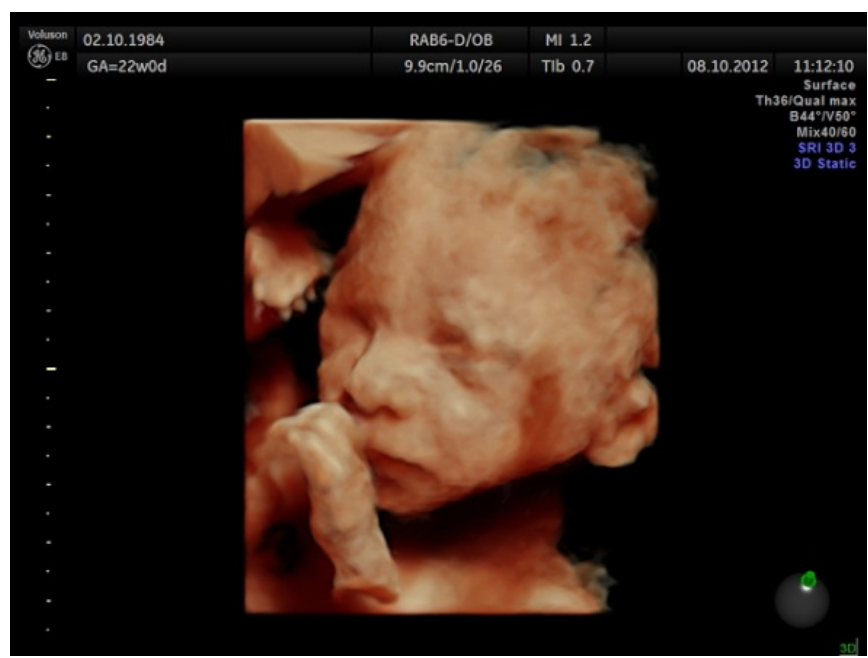

Figure 3. Fetus at 22 weeks pm. 


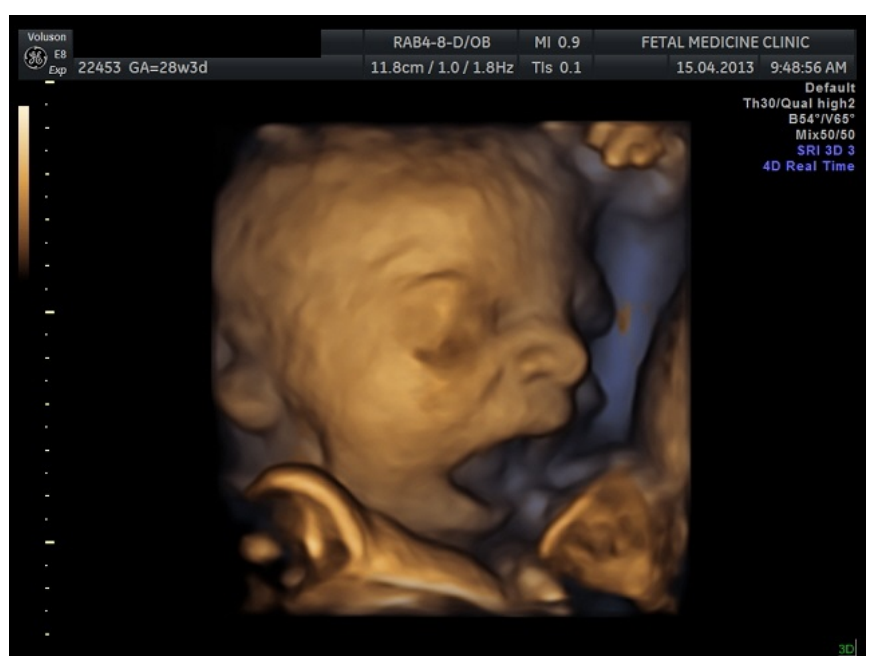

Figure 4. Fetus at 28 weeks pm.

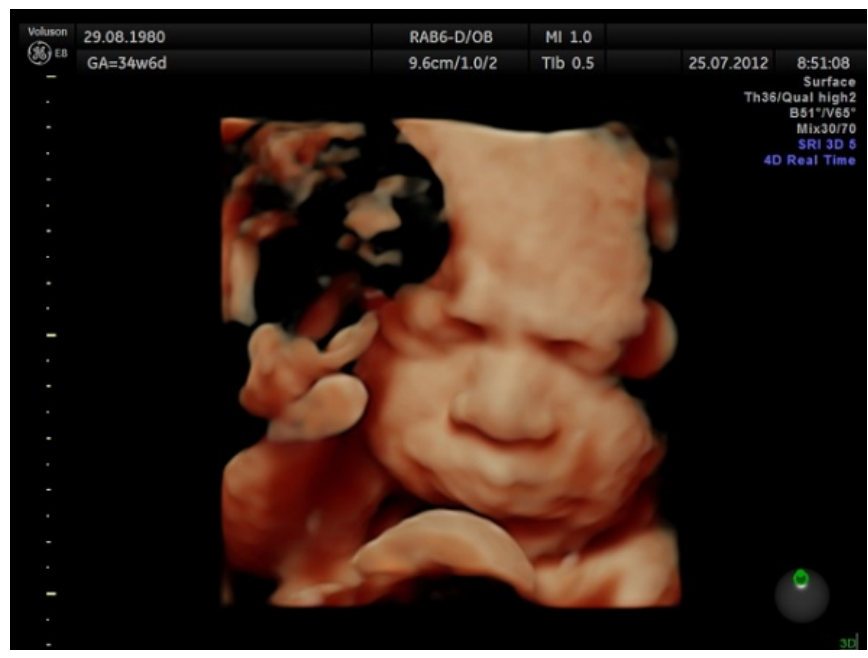

(a)

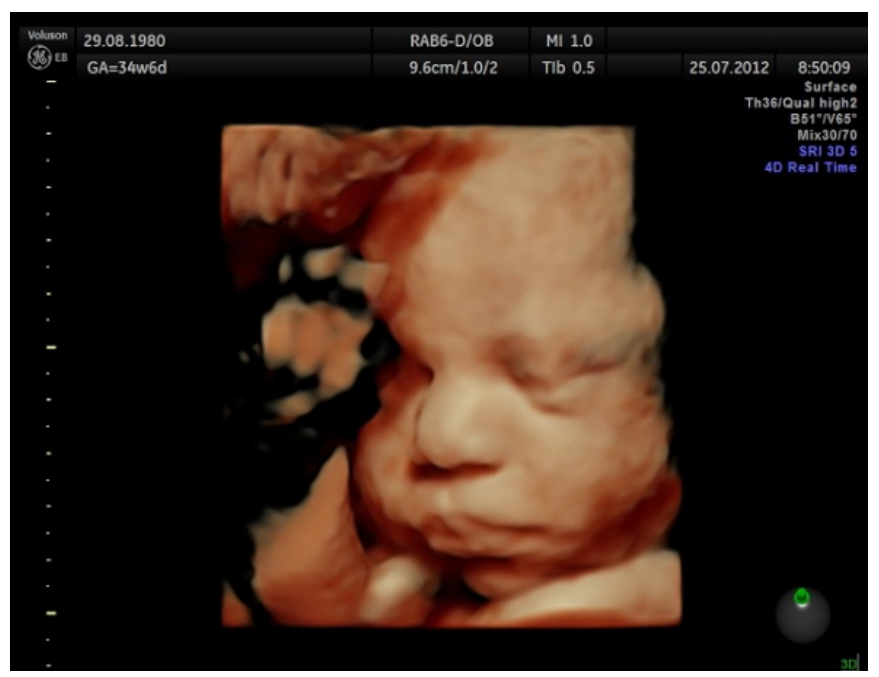

(b)

Figure 5. (a) Fetus at 35 weeks pm. (b) Fetus at 35 weeks pm. 
ultrasound in this specific clinical constellation.

A more subtle form of "maternal absence during pregnancy" with its implications for different forms of maternal communication with the fetus like speaking, singing, touching and holding of the womb, could be seen in prenatal illicit drug abuse. Substance use during pregnancy severely reduces a woman's ability of transition into the emotional achievements of motherhood, a task vital for a successful bonding process and loving relationship with the fetus (Rubin, 1984). There are assumptions that low levels of prenatal attachment could be related to forms of fetal- and child abuse. So far, only two studies have evaluated this correlation, which indicates the necessity to further research this phenomenon (Laxton-Kane, 2002; Pollock, 1999). 3D/4D ultrasound with the potential to improve fetal-maternal attachment, in cooperation with a clinical psychologist, could be a tool for therapeutic intervention in these cases.

\section{Maternal Loneliness}

We are reporting the case of a 40 year old 2nd gravida, 1 para, with spontaneous pregnancy. This lady had an uneventful previous medical and obstetrical history of an uncomplicated first pregnancy, with normal vaginal delivery at term. In this pregnancy she had normal pregnancy surveys with normal ultrasound (US) scans at 6 and 8 weeks. She had a low risk evaluation of her 1st trimester nuchal translucency screening, and a normal morphology scan at 20 weeks. Though she changed her obstetrician thrice until 26 weeks, she adhered to regular checkups with her obstetricians every 4 weeks. She felt fetal movements first at 18 weeks and emphasized that they were more frequent and vigorous than in her first pregnancy where she started to notice fetal movements only at 21 weeks. At 26 weeks of the reported pregnancy, fetal movements stopped suddenly and almost completely within 24 hours. She went to an emergency unit where she was reassured. At 30w2d she was referred for Doppler scan and fetal biometry. In US normal morphology was detected. Biometry corresponded to 28w1d. Abdominal circumference minus 2.4 SD, cerebellum diameter minus 2.2 SD indicated moderate intrauterine growth restriction (IUGR). Apparently normal neuro-sonoanatomy with normal ventricular width was noticed. Normal Doppler flow parameters of fetal (umbilical artery, middle cerebral artery) and maternal side (uterine arteries) and moderate polyhydramnios were observed. Outstanding observation was the permanent immobility of the entire fetus in 4D-US. The fetus was in breech position with extended legs, the tongue was protruding from a slightly gaping mouth with moderate retrognathia, and the fists were clenched. We found during our two 4D-US examinations an abnormal Kurjak Antenatal Neurodevelopmental Test (KANET) score, the first at 30 w2d with a score of 3 points, and the second at 31w4d, with a score of 4 points. Cardiotocography (CTG) demonstrated complete loss of variability and accelerations, in repeated CTG samples with registration over more than 30 minutes each. (Image 6) We discussed the abnormal findings with the parents and suggested amniocentesis for karyotyping which was carried out at 30w3d. The cytogenetic report confirmed 46,XX without numerical and structural chromosomal anomalies. Parvovirus-PCR in amniotic fluid was negative. Acetylcholinesterases and alpha-fetoprotein levels in amniotic fluid were normal. At 32 weeks, our patient decided to travel to the USA for delivery. There, she developed at 33 weeks severe polyhydramnios with breathing difficulties, back pain and sleep disorder. At 34 weeks, 3.5 liters of amniotic fluid were drained per amniocentesis. The procedure was followed by onset of labor. The preterm female baby was born at 34w2d through low transverse C-section because of breech position. The baby was delivered without cries or respiration and successfully intubated on third attempt at 17 minutes post delivery and continued on positive pressure ventilation. The clinical manifestations of severe contractures, the Pena-Shokeir phenotype and the akinesia indicated the possibility of neurologic disturbance. The possibility of an intrauterine insult was considered more likely than a genetic syndrome. Postnatal brain MRI showed global volume loss of hemispheres and cerebellum, EEG diffuse low voltage and slow activity consistent with severe encephalopathy, and US demonstrated findings of bilateral intraventricular hemorrhage and increased echogenicity of bilateral basal ganglia. After extensive discussion of the catastrophic brain damage with the parents, the baby was compassionately extubated on day 5 post partum and passed away 30 minutes after extubation. The parents requested an autopsy. In the process of retrospective case evaluation, we experienced the mother as full of trust and very cooperative. She was able to speak about "the baby which had stopped moving". Appropriate counseling and mental preparation of the parents for an unfavorable pregnancy outcome was based on four dimensional ultrasound (4D) evaluations of the fetus, showing repeated abnormal KANET and revealing the true extent of fetal compromise by means of understandable 4D video sequences. After seven months the patient was pregnant again and came for ultrasound 2nd trimester scan (Honemeyer, 2013; Kurjak, 2010) (Figure 6). 


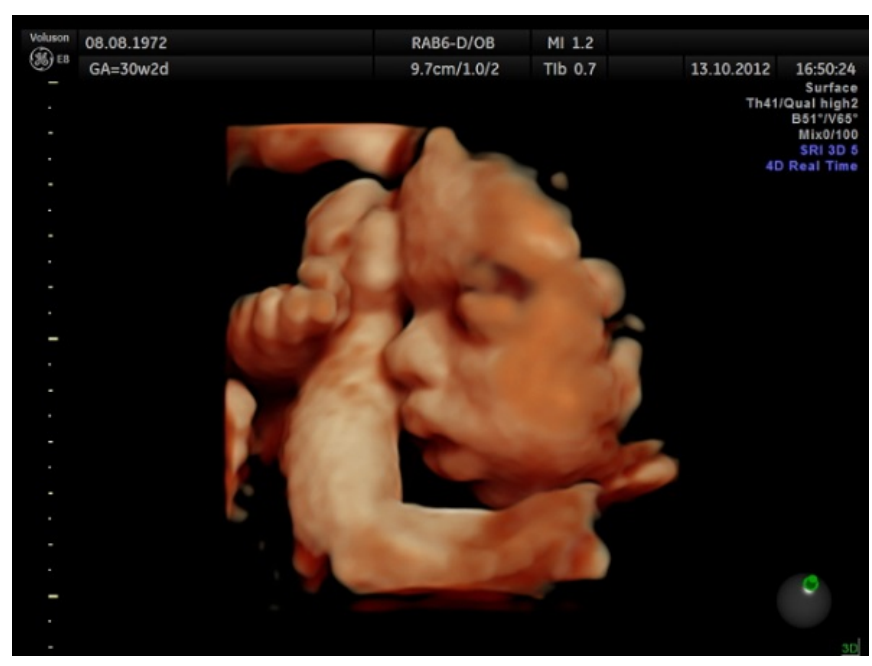

Figure 6. Immobile fetus at 31 weeks.

Families can be profoundly disturbed by the traumatic event of a fetal loss. When experiencing such a loss parents often grieve for many years, and the next pregnancy might rouse anxieties which could negatively affect fetal-parental attachment. The diagnosis of a serious fetal abnormality and/or fetal or perinatal death can be a devastating experience especially if such abnormalities have no pre- and postnatal treatment option (Kenner, 2000). Freud proposed that the mourning process involved freeing oneself from attachments to the dead person, releasing oneself from inhibitions about becoming a separate being from the dead person, and resolving conflicts of ambivalence over the lost love relationship (Freud, 1917).

The value of 3D/4D ultrasound as supportive measure in grief work (Maier, 1998; Clower, 2003) in such situations is twofold:

1) Diagnosis of fetal malformation often remains abstract for parents even after extensive explanation, and in their confusion they find it difficult to accept the facts in mere verbal form: one 3D image however can explain findings better than thousand words, because "to see is to believe". 3D/4D provides high quality images of the embryo and fetus and gives couples the possibility to sensually grasp image information and "understand" sonographic findings of malformation.

2) In a following normal pregnancy, 3D/4D ultrasound can demonstrate the integrity of the fetus by showing face, body, and normal movements, thus countering negative parental expectations and anxieties of recurrence.

\section{Conclusion}

It is only within the past 30 years that visualizing of the living fetus has been possible. Previously, fetal life was often acknowledged once "quickening" developed, when the pregnant woman first felt her baby moving within her uterus. The option and ability to view the fetus as an independent being at an earlier point in pregnancy likely contributes to the maternal-fetal relationship developing at a much earlier point in fetal development (Stormer, 2003). Studies measuring Maternal-Fetal Attachment (MFA) prior to the performance of any type of ultrasound have delivered evidence that MFA was increased based upon having an ultrasound (Righetti, 2005). 3D/4D ultrasound can be used as a therapeutic element not only in preparing fetal-parental bonding, but even more in situations where the harmonic interdependent psycho-physical development of mother/father and fetus is disturbed.

\section{References}

Alhusen, J. L. (2008). A Literature Update on Maternal-Fetal Attachment. Journal of Obstetric, Gynecologic, \& Neonatal Nursing, 37, 315-328. http://dx.doi.org/10.1111/j.1552-6909.2008.00241.x

Bilko, A., Altbaecker, V., \& Hudson, R. (1994). Transmission of Food Preference in the Rabbit: The Means of Information Transfer. Physiology \& Behavior, 56, 907-912. http://dx.doi.org/10.1016/0031-9384(94)90322-0

Castiello, U., Becchio, C., Zoia, S., Nelini, C., Sartori, L. et al. (2010). Wired to Be Social: The Ontogeny of Human Interac- 
tion. PLoS ONE, 5, e13199. http://dx.doi.org/10.1371/journal.pone.0013199

Clark, A. (2008). Supersizing the Mind: Embodiment, Action, and Cognitive Extension. Oxford: Oxford University Press, $286 \mathrm{pp}$.

Clower, C. E. (2003). Pregnancy Loss: Disenfranchised Grief and Other Psychological Reactions. Doctor of Philosophy (Clinical Psychology), Denton, TX: University of North Texas, 136 p.

Cranley, M. S. (1981). Development of a Tool for the Measurement of Maternal Attachment during Pregnancy. Nursing Research, 30, 281-284. http://dx.doi.org/10.1097/00006199-198109000-00008

Esmaeilzadeh, M., Dictus, C., Kayvanpour, E. et al. (2010). One Life Ends, Another Begins: Management of a Brain-Dead Pregnant Mother-A Systematic Review. BMC Medicine, 8, 74. http://dx.doi.org/10.1186/1741-7015-8-74

Eswaran, H. (2002). Magnetoencephalographic Recordings of Visual Evoked Brain Activity in the Human Fetus. The Lancet, 360, 779-780. http://dx.doi.org/10.1016/S0140-6736(02)09905-1

Fleming, A. S., Ruble, D. N., Flett, G. L., \& Shaul, D. L. (1988). Postpartum Adjustment in First-Time Mothers: Relations between Mood, Maternal Attitudes, and Mother-Infant Interaction. Developmental Psychology, 24, 71-81. http://dx.doi.org/10.1037/0012-1649.24.1.71

Freud, S. (1959). Mourning and Melancholia. Collected Papers. New York: Basic Books, 152-170 (original work published 1917).

Graven, S. N, \& Browne, J. V. (2008). Auditory Development in the Fetus and Infant. Elsevier Inc., Amsterdam.

Honemeyer, U., Kasirsky, J., Pour-Mirza, A., \& Kurjak, A. (2013). KANET Diagnosis of Fetal Akinesia Deformation Sequence at 30 Weeks. Donald School Journal of Ultrasound in Obstetrics and Gynecology, 7, 500-505. http://dx.doi.org/10.5005/jp-journals-10009-1324

Kandel, D. B., Wu, P., \& Daies, M. (1994). Maternal Smoking during Pregnancy and Smoking by Adolescent Daughters. American Journal of Public Health, 84, 1407-1413. http://dx.doi.org/10.2105/AJPH.84.9.1407

Kenner, C., \& Dreyer, L. A. (2000). Prenatal and Neonatal Testing and Screening: A Double-Edged Sword. Nursing Clinics of North America, 35, 627-642.

Kirstein, C. L., Philpot, R. M., \& Dark, T. (1997). Fetal Alcohol Syndrome: Early Olfactory Learning as a Model System to Study Neurobehavioral Deficits. International Journal of Neuroscience, 59, 119-132. http://dx.doi.org/10.3109/00207459708988467

Klaus, M. H., Kennell, J. H., \& Klaus, P. H. (1995). Bonding: Building the Foundations of Secure Attachment and Independence. In M. H. Klaus, J. H. Kennell, \& P. H. Klaus (Eds.), Menlo Park, CA: Addison-Wesley.

Kurjak, A., Abo-Yaqoub, S., Stanojevic, M., Yigiter, A. B., Vasilj OLebit, D., Shaddad, A. N., Ahmed, B., Kavak, Z. N., Miskovic, B. et al. (2010). The Potential of 4D Sonography in the Assessment of Fetal Neurobehavior-Multicentric Study in High-Risk Pregnancies. Journal of Perinatal Medicine, 38, 77-82. http://dx.doi.org/10.1515/jpm.2010.012

Laxton-Kane, M., \& Slade, P. (2002). The Role of Maternal Prenatal Attachment in a Woman's Experience of Pregnancy and Implications for the Process of Care. Journal of Reproductive and Infant Psychology, 20, 253-266. http://dx.doi.org/10.1080/0264683021000033174

Lecanuet, J. P., \& Schaal, B. (2002) Sensory Performances in the Human Foetus: A Brief Summary of Research. Intellectica, 34, 29-56.

Lecanuet, J.-P., Granier-Deferre, C., Jacquet, A. Y., Capponi, I., \& Ledru, L. (1993) Prenatal Discrimination of a Male and Female Voice Uttering the Same Sentence. Early Development and Parenting, 2, 217-228. http://dx.doi.org/10.2105/AJPH.84.9.1407

Maier, B. et al. (1998). Psychological Influence of Three-Dimensional Fetal Imaging on Women with High-Risk Pregnancies. In E. Merz (Ed.), 3D-Ultrasound in Obstetrics and Gynecology (pp. 149-152). Philadelphia, PA: Lippincott Williams \&Wilkins.

Mellor, D. J., Diesch, T. J., Gunn, A. J., \& Bennet, L. (2007) Fetal “Awareness” and “Pain”: What Precautions Should Be Taken to Safeguard Fetal Welfare during Experiments? Proceedings of the 6th World Congress on Alternatives \& Animal Use in the Life Sciences, 21-25 August 2007, Tokyo, 79-83.

Moore, J. K., \& Linthicum, F. H. (2007). The Human Auditory System: A Timeline of Development. International Journal of Audiology, 46, 460-478.

Muller, M. E. (1996). Prenatal and Postnatal Attachment: A Modest Correlation. Journal of Obstetric, Gynecologic, and Neonatal Nursing, 25, 161-166. http://dx.doi.org/10.1111/j.1552-6909.1996.tb02420.x

Pollock, P. H., \& Percy, A. (1999). Maternal Antenatal Attachment Style and Potential Fetal Abuse. Child Abuse and Neglect, 23, 1345-1357. http://dx.doi.org/10.1016/S0145-2134(99)00101-5

Righetti, P. L., Dell’Avanzo, M., Grigio, M., \& Nicolini, U. (2005). Maternal/Paternal Antenatal Attachment and Fourth- 
Dimensional Ultrasound Technique: A Preliminary Report. British Journal of Psychology, 96, 129-137. http://dx.doi.org/10.1348/000712604X15518

Rubin, R. (1984). Maternal Identity and the Maternal Experience. New York: Springer.

Schaal, B., Marlier, L., \& Saussignan, R. (2000) Human Foetuses Learn from Their Pregnant Mothers Diet. Chemical Senses, 25, 729-737. http://dx.doi.org/10.1093/chemse/25.6.729

Smotherman, W. P., \& Robinson, S. R. (1991) Odour Aversion Learning by the Rat Fetus. Physiology \& Behavior, 29, 769-771. http://dx.doi.org/10.1016/0031-9384(82)90322-5

Stormer, N. (2003). Seeing the Fetus: The Role of Technology and Image in the Maternal-Fetal Relationship. Journal of the American Medical Association, 289, 1700. http://dx.doi.org/10.1001/jama.289.13.1700

von Hofsten, C. (2009). Action, the Foundation for Cognitive Development. Scandinavian Journal of Psychology, 51, 1-7. 